\title{
Superconducting Solenoid Lens for a High Energy Part of a Proton Linac Front End
}

\author{
E. Joseph DiMarco, Emil Khabiboulline, Darryl F. Orris, Michael A. Tartaglia, Iouri Terechkine
}

\begin{abstract}
Development of a high current proton linac at FNAL went through many stages, starting from a pulsed $8 \mathrm{GeV}$ linac, then to the HINS linac front end R\&D, and now moves toward the ProjectX CW linac. For different parts of the accelerator front end in each of these linacs, the design requires solenoid-based focusing lenses that can provide the needed transverse focusing in a very tight real estate environment. The multiple, often contradictory, design requirements of focusing lenses include the need of high focusing strength, small footprint, very low fringe field, and embedded steering coils. To meet these requirements, a series of prototype lenses were built and tested. Performances of the lenses designed for low energy parts of the linac front end have been reported earlier. This report presents lens design and test data for the high energy part of a proton linac front end, up to an energy of $\sim 100 \mathrm{MeV}$. For these lenses, reliable protection from high voltages or temperatures during a quench becomes important, and a new protection scheme was developed which allows more flexibility and reliability. Details of the magnetic axis position have also been investigated.
\end{abstract}

Index Terms-Superconducting Magnet, Solenoid, Quench Protection, Modeling

\section{INTRODUCTION}

A series of focusing solenoids for a high intensity R\&D proton linac front end have been designed and built at Fermilab. In this program known as HINS (for High Intensity Neutrino Source), solenoid focusing optics are used to control beam emittance and halo growth up to an energy of $100 \mathrm{MeV}$. The focal length is proportional to the integral $\left(\int \mathrm{B}_{\mathrm{z}}{ }^{2} \mathrm{dz}\right)^{-1}$, where $\mathrm{z}$ is the axial direction. $\mathrm{B}_{\mathrm{z}}$ must increase roughly as the square root of the beam kinetic energy (linearly with velocity $\beta)$ to maintain a constant focusing period. In the HINS design, accelerating structures and superconducting focusing elements are combined in three sections of increasing beam energy: from 2.5 to $10 \mathrm{MeV}$, " $\mathrm{CH}$ " solenoids alternate with room temperature "Crossbar-H" copper cavities; from 10 to $30 \mathrm{MeV}$, "SS1" solenoids alternate with Superconducting Single Spoke Resonator (SSR) cavities in two long

Manuscript received October 9, 2012. This work was supported in part by Fermi Research Alliance, LLC, under contract No. DE-AC02-07CH11359 with the U.S. Department of Energy.

E. J. DiMarco, D. F. Orris, M. A. Tartaglia and I. Terechkine are with the Fermi National Accelerator Laboratory, Batavia IL 60510 USA (corresponding author phone: 630-840-3890; fax: 630-840-8079; e-mail: tartaglia@fnal.gov).

E. Khabiboulline is a student at California Institute of Technology, Pasadena, CA; email: ekhabibo@caltech.edu. cryomodules; and from 30 to $100 \mathrm{MeV}$ "SS2" solenoids alternate with SSR cavities in two cryomodules [1]. Reference [2] contains an overview of the design constraints and solenoid R\&D challenges. Progress in developing these lenses has previously been reported for the $\mathrm{CH}$ and $\mathrm{SS} 1$ solenoids [3-8]. In this paper we discuss the design and performance of a model SS2 solenoid and make detailed comparisons of the magnet quench performance to predictions of a model used to study quench development and protection.

\section{SS2 SOLENOID DESIGN}

The basic design considerations for SS2 are similar to those for the SS1 solenoid [9], in terms of: aperture, steering dipole correctors, desired operating margin, field cancellation at the ends using bucking coils, and a flux clamping iron yoke. SS1 lenses require a field-squared integral $\mathrm{B}^{2} \mathrm{~L}=3.0 \mathrm{~T}^{2}-\mathrm{m}$, while $\mathrm{SS} 2$ requires $\mathrm{B}^{2} \mathrm{~L}=5.0 \mathrm{~T}^{2}-\mathrm{m}$. Assuming the same high quality $\mathrm{NbTi}$ strand and packing factor as SS1 is obtained, to increase $\mathrm{B}^{2} \mathrm{~L}$ for $\mathrm{SS} 2$ essentially requires a longer main coil length. The increase in stored energy also scales with $\mathrm{B}^{2} \mathrm{~L}$, therefore the main concern is protection of the coils from high voltages and temperatures that may develop during a quench.

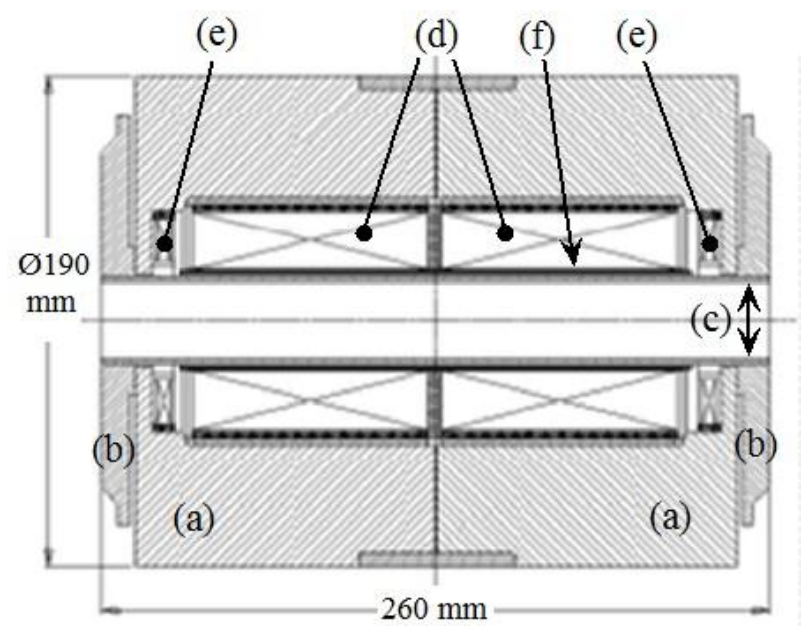

Fig. 1. The SS2 solenoid design cross section: a) soft iron flux return yoke, b) end flange, c) stainless steel beam tube, d) main coil, e) bucking coil, f) thin steering dipole coils. Outer dimensions shown are in $\mathrm{mm}$.

The SS2 cross section is shown in Fig. 1. In the SS2 design [10], the "main coil" was split into two separate coils separated by an insulating wall. As a practical matter they can be more easily fabricated than one long coil. Although it adds an extra pair of leads, this feature allows more degrees of 
freedom for quench detection and protection, as discussed later in detail.

The SS1 and SS2 designs use the simplest configuration in which the main coils (MC) and bucking coils (BC) are in series and at the same current. Early modeling studies showed that the most dangerous case is for a high current quench in a $\mathrm{BC}$, where all of the solenoid's stored energy may be dissipated in a small volume wound with narrow $(0.5 \mathrm{~mm}$ round) strand. The early quench development model [11] and test results [8] demonstrated that the SS1 was relatively safe when using a single optimized dump resistor across the power leads for energy extraction.

The design of SS2 main coils utilized a larger cross section rectangular $(1.0 \mathrm{~mm} \times 0.6 \mathrm{~mm})$ strand operated at higher current than was used in the SS1 $(0.8 \mathrm{~mm}$ round). This was done to obtain a higher packing factor and reduce inductance. Initial modeling of the SS2 quench case (which assumed a single long MC) was made to determine the optimum dump resistor [11]. It found that the $\mathrm{BC}$ voltage to ground would reach $\sim 600 \mathrm{~V}$ and temperature would rise up to $240 \mathrm{~K}$ at the maximum quench current. To address this concern, a new quench modeling study was made to predict the behavior of temperatures and voltages under various quench scenarios, and to optimize the combination of resistors across the coils for protection. Subsequently, the prototype SS2 solenoid was tested and a detailed comparison of the coil voltage development was made for each of the scenarios.

\section{QUENCH DEVELOPMENT MODEL}

A computational tool for modeling quench propagation was developed at Fermilab specifically to study protection issues with superconducting solenoids for HINS [12]. The model solves the $2 \mathrm{D}$ problem of heat and quench propagation in the radial and longitudinal directions, which were shown for this case to exceed the propagation rate along the strand [13]. The original code has evolved to include a convenient user interface for making changes to the many solenoid parameters, geometry, material properties, and quench origins. The improved code allows for input of magnetic field maps and coil self- and mutual-inductances [14].

For the new SS2 quench study, the main coil was split into two coils (to match the as-built design). Additional capabilities were added to allow the placement of resistors across each individual coil and to initiate the development of quenches in multiple coils at different times [15]. This latter feature is necessary to reflect the behavior observed in the actual quench tests, because the model does not consider eddy current heating during the rapid current discharge, which might induce a secondary quench.

Simulation of quench development was then made for several configurations of external protection resistors: a) a single dump resistor in parallel with the solenoid [14], b) dump resistors across either both BCs, or both MCs [16], c) dump resistors in parallel with each of the coils [17]. A systematic study was made to determine the optimum resistor values in each case, to minimize the peak temperature and voltage to ground. In case (a) the optimum dump resistor was found to be $2.9 \Omega$; in case (b) the optimal case is $1.3 \Omega$ across each MC; in case (c) the best arrangement is $1 \Omega$ connected in parallel with each MC and $3 \Omega$ connected in parallel with each BC.

\section{Test OVERVIEW AND QUENCH PERFORMANCE}

The SS2 solenoid was tested at the Fermilab magnet test facility stand 3, a vertical dewar, in a $4.4 \mathrm{~K}$ helium bath [18]. The four coils were all spliced in series inside the vessel, but each superconducting lead and each splice were connected to a vapor-cooled current lead. Thus, with 5 leads it was possible to connect an external dump resistor in parallel with each coil of the solenoid, or to connect a dump resistor across the entire magnet. During the test, each of the three optimized resistor configurations was implemented to capture voltage and current data for comparison with simulation results. In addition, another case (d), with no dump resistor, was inadvertently tested (due to an incorrectly programmed delay of the dump switch).

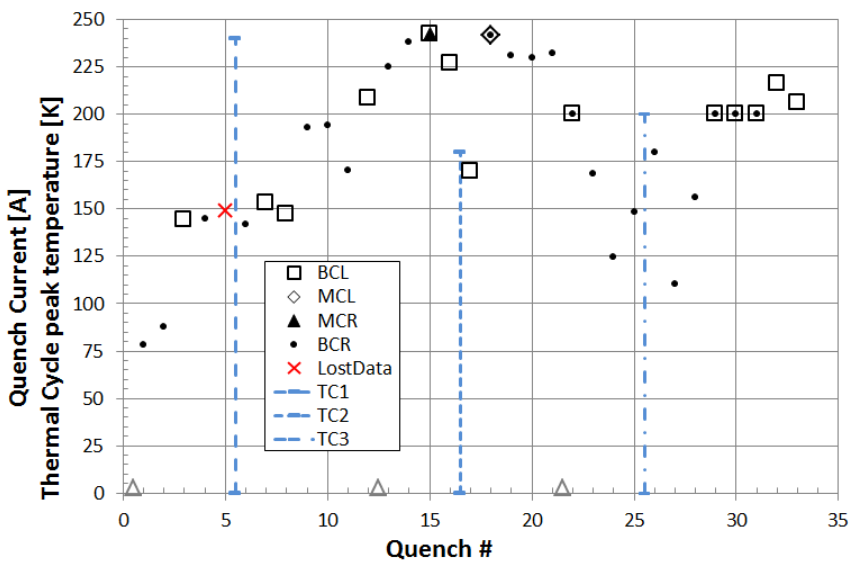

Fig. 2. Quench training history of the SS2 solenoid, showing quench current versus quench, and intervening partial thermal cycles. Open triangles on the horizontal axis indicate changes of the protection resistor configuration. Points with multiple symbols indicate quench started in multiple coils, although not necessary at the same starting time, before quench detection.

Hall probe magnetic measurements confirmed that the field profile agreed with the expected magnitude and shape. Fig. 2 shows the quench history of the SS2 solenoid; coils are labeled by location, " $L$ " on the lead end, " $\mathrm{R}$ " on the non-lead or "return" end. There were several partial thermal cycles (TC) as the magnet and test dewar were allowed to warm during weekends. Quenches at 200 A occurred while testing the steering dipole coils with the solenoid at its nominal operating current. Some slight retraining is evident after each TCs. Once the magnet reached the expected maximum quench current at 240 A, it showed some erratic behavior. This is likely explained by a loss of pre-stress allowing coil motion: it was found that some of the bolts used to hold the yoke halves together had worked loose. For a reliable magnet, future versions of the design must include measures to prevent this.

The initial resistor configuration tested in quenches 1-12 was (b) with $1.3 \Omega$ across each MC. Quenches 13-21 used 
configuration (c) with $3 \Omega$ across each BC and $1 \Omega$ across each MC. After quench 21 , a $3 \Omega$ dump resistor was installed across the entire coil, but the dump switch timing was not corrected immediately; thus data were captured for case (d) up to $185 \mathrm{~A}$ and case (a) up to $216 \mathrm{~A}$. Note that MC quenches only occurred at the maximum quench current and $\mathrm{BCs}$ also quenched. Our principal focus is on the $\mathrm{BC}$ quench case, and Fig. 3 shows how the measured peak $\mathrm{BC}$ voltage depends upon quench current, for case (b); the linear behavior is typical of all cases, although the slope changes. Clearly there is a threshold current for a second BC quench, and at high current the second quench helps to dissipate energy more uniformly than in a single quenching $\mathrm{BC}$.

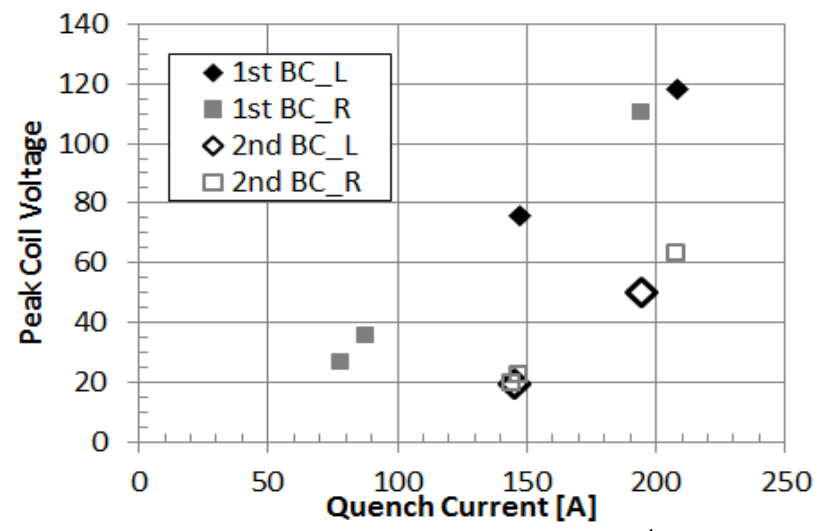

Fig. 3. Measured peak coil voltage in the $1^{\text {st }}$ and $2^{\text {nd }}$ quenching $B C$, identified by which $\mathrm{BC}$ quenched $1^{\text {st }}$ or $2^{\text {nd }}$, for dump resistor case (b) events. At low current, no $2^{\text {nd }}$ quench develops. (Two $1^{\text {st }} \mathrm{BC}$ points are not shown because of ADC saturation early in the test; gains were then lowered).

\section{TEST AND MOdEL COMPARISON}

A detailed study of the coil voltage development, current discharge profile, and temperature rise was performed for each resistor configuration tested. Comparisons of the voltage and current profiles with actual data were made [18]. In events where a second $\mathrm{BC}$ quench is seen to start, this is also simulated. An example of the predictions and model comparison for case (c) are shown in Fig. 4 (current, temperatures), and Fig. 5 (voltages). Note that the BCR voltage starts negative (inductive), then grows resistive.

The qualitative trend in all cases is that the model predicts faster initial current decay and larger voltage growth at earlier time than are seen in the data. In fact the model follows the same trends shown for data in Fig. 3 but predicts higher coil voltages by a factor that depends on dump resistor case. A summary of the comparison is given in Table I, where the average data/model ratio of peak voltages is shown, for three quench events of each case studied.

In most cases the actual voltages are about .6 to .75 those predicted. Although in case (d) the $2^{\text {nd }} \mathrm{BC}$ measured quench voltages are slightly greater than what the model would suggest, they are also much lower than for the other cases. Also, in case (a) the model sharply overestimates actual BC and MC voltages: Fig. 6 shows the actual and predicted voltages for this case. They suggest both MCs quench, but the

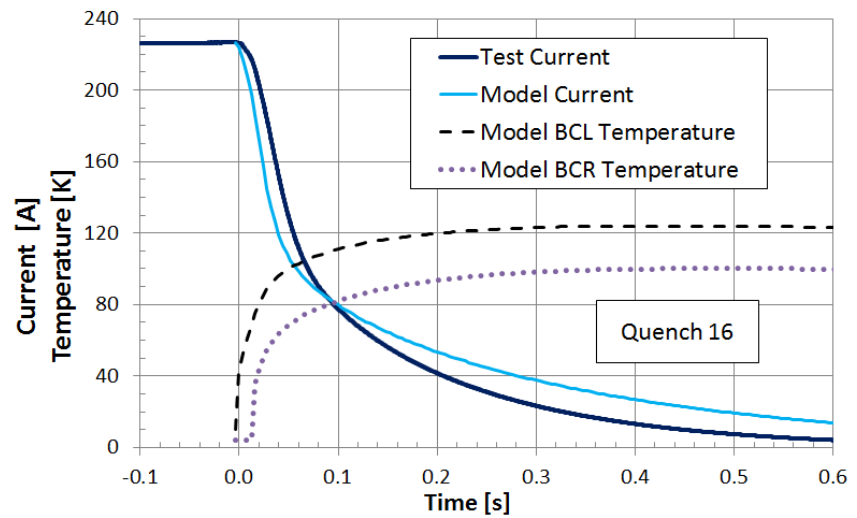

Fig. 4. Measured and predicted current decay, and predicted temperature profiles following a quench with dump resistor configuration (c). Quench detection occurs at $\mathrm{t}=0$.

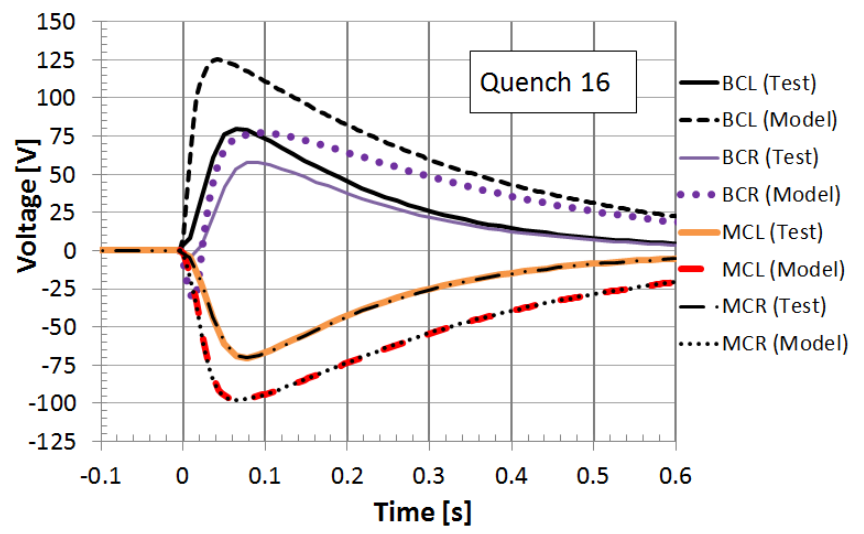

Fig. 5. Measured and predicted coil voltage profiles following a BCL quench with dump resistor configuration (c). Note that the MCL and MCR profiles are identical for both data and model (same inductance, no quench).

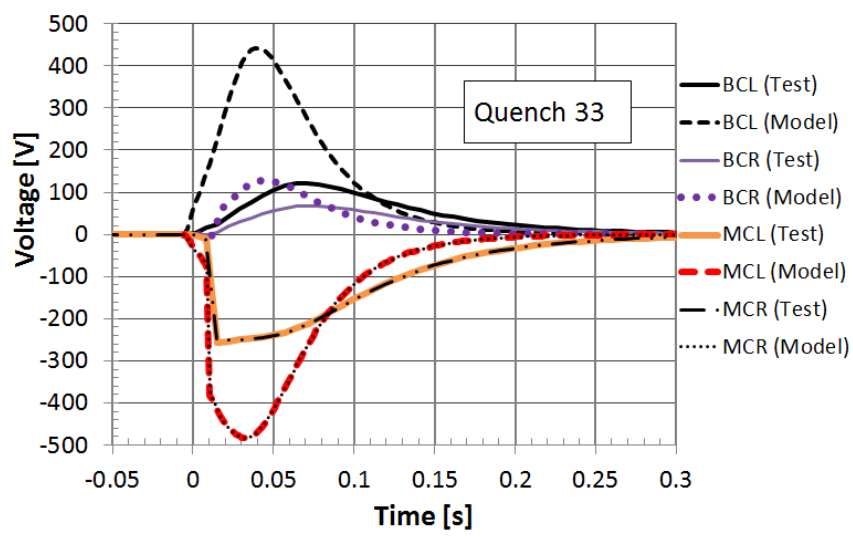

Fig. 6. Measured and predicted coil voltage profiles for a case (a) BCL quench with a $3 \Omega$ dump resistor across the solenoid leads.

model comparison includes this effect. This case is not yet explained; an instrumentation issue is not yet ruled out.

TABLE I DATA/MODEL PeAK VolTage COMPARISON

\begin{tabular}{cccc}
\hline \hline Case & $\mathbf{1}^{\text {st }} \mathbf{B C}$ & $\mathbf{2}^{\text {nd }} \mathbf{B C}$ & $\mathbf{M C}$ \\
\hline $\mathrm{a}$ & 0.30 & 0.46 & 0.55 \\
$\mathrm{~b}$ & 0.64 & 0.78 & 0.70 \\
$\mathrm{c}$ & 0.61 & 0.75 & 0.71 \\
$\mathrm{~d}$ & 0.62 & 1.25 & 0.66 \\
\hline \hline
\end{tabular}


We can conclude that the model is conservative in all of these cases as voltage and temperature predictions are higher than actually developed in the SS2 coils. Both data and models suggest that case (c) is the safest configuration of resistors to use. The predicated peak temperature versus predicted peak voltage is shown in Fig. 7 for all cases; the measured peak $\mathrm{BC}$ voltages are overlaid to get a sense of what the actual peak temperatures might be by comparing to the model envelope.

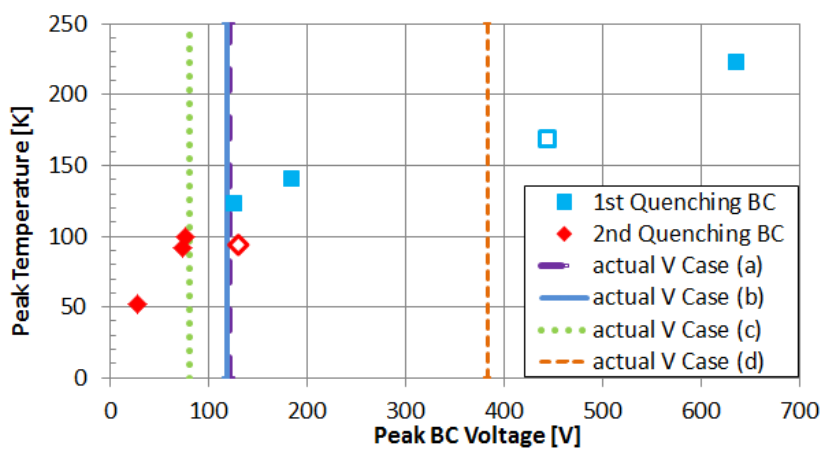

Fig. 7. Predicted peak temperature versus peak voltage for the four simulated dump resistor cases at the highest quench current for each; open symbols are case (a) model points, and data for the $1^{\text {st }} \mathrm{BC}$ are indicated.

Some possibilities for further improvement of the model can be imagined, and these test data will be a benchmark for checking predictions that incorporate further developments. Self- and mutual- inductances are now calculated in detail for the static case, dynamic effects will reduce them; these inductances could be measured. Thermal conductivities of the composite materials (strand) could be better approximated. The true 3D model could also be implemented.

\section{MAGNETIC FIELD AND ALIGNMENT}

The solenoid field shape was mapped at the nominal $200 \mathrm{~A}$ operating current after quench 21, coarsely and with fine end field scans. Fig. 8 shows the agreement is very good between data and model for the main field, less so for the fringe fields. In an attempt to measure its shielding effect on the fringe field, a pure $\mathrm{Nb}$ sheet was placed just outside the return end during the test. The model comparison shown is without this

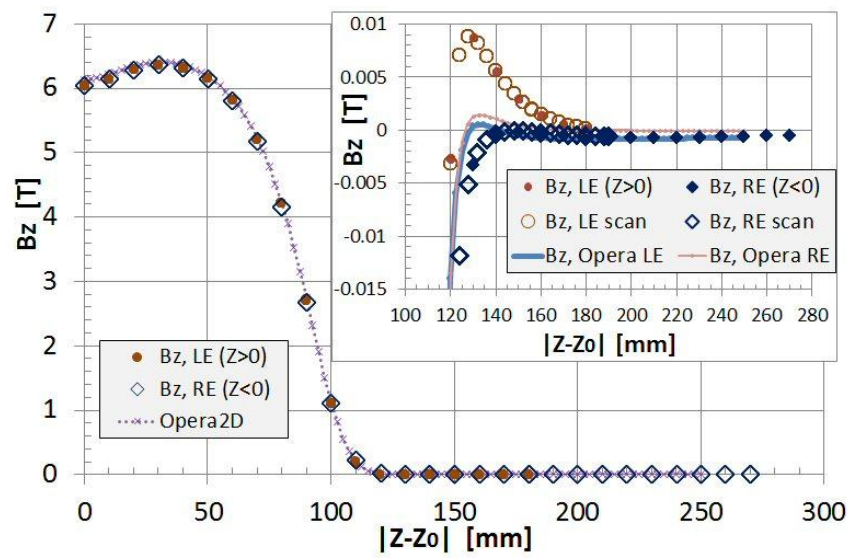

Fig. 8. Predicted and measured axial field profiles for the SS2 solenoid, as a function of distance from the center; insert shows the fringe field details for both ends, compared to the as-built model. Effect of $\mathrm{Nb}$ shield at RE has not been included in the model prediction. contribution. Further study is needed to understand if the difference in shapes at this end is due to $\mathrm{Nb}$ shielding, or $\mathrm{BC}$ position errors perhaps resulting from loosened bolts.

The steering dipole field was also mapped, with both $\mathrm{H}$ and $\mathrm{V}$ correctors powered in series at $100 \mathrm{~A}$. The maximum (quench) current is $47.8 \mathrm{~A}$ with the solenoid at $200 \mathrm{~A}$; at this current each coil has an integrated bend strength of $1.5 \mathrm{~T}-\mathrm{cm}$.

The solenoid magnetic axis position continues to be of interest, especially with four coils that might each be offset by some amount, and may change during construction phases or operating conditions. Systematic measurements with Stretched Wire of the SS2 axis were made after initial assembly, before and after beam tube welding. They are yet to be repeated after cold testing, but since bolt torques were found to have relaxed there is still discussion how to proceed.

\section{CONCLUSION}

A prototype SS2 focusing solenoid for the high energy part of the HINS proton linac front end was built and tested, and results were subjected to detailed comparison with model predictions of quench and magnetic performance. The latter are in good general agreement, but the fringe field region is not yet fully understood. Quench development was carefully analyzed under four different protection resistor configurations: the actual voltages were lower and developed more slowly than predicted, and the solenoid survived quenches without protection - although it reached nearly 400 $\mathrm{V}$ to ground in the bucking coil. Though somewhat conservative quantitatively, in general the model reflects the data well qualitatively. Some additional improvements are envisioned that can be implemented and checked with these data. Continued development and use of this computational tool will be valuable as Fermilab moves from the HINS program into the needed R\&D for a ProjectX linac front end, which will also utilize solenoids with large stored energy [19].

\section{ACKNOWLEDGMENT}

The programmatic support over many years to design, construct, and test the HINS series of solenoids is very gratefully acknowledged, along with the skilled technical assistance from Magnet Systems and Test Department personnel at Fermilab to build the solenoids and test them. We appreciate the strong commitment to support numerous talented students who have contributed significantly to the modeling, test, analysis and documentation efforts.

\section{REFERENCES}

[1] P. Ostroumov, B. Mustapha, V. N. Aseev, "Beam Dynamics Studies of the 8-GeV Superconducting $\mathrm{H}^{-}$Linac," Proceedings of LINAC 2006, TUP072, 2006, pp420-423.

[2] G. Apollinari, et al., "HINS Linac Front End Focusing System R\&D," IEEE Trans. Appl. Supercon., Vol. 19, No. 3, June 2009, pp.1368-1371.

[3] R. H. Carcagno, et al., "Superconducting Solenoid Magnet Test Results," IEEE Trans. Appl. Supercon., Vol. 17, No. 3, June 2007, pp.1213-1216.

[4] M. Tartaglia, et al., "Test Results for HINS Focusing Solenoids at Fermilab," IEEE Trans. Appl. Supercon., Vol. 19, No. 3, June 2009, pp.1352-1355. 
[5] T. Page, et al., "HINS Superconducting Lens and Cryostat Performance," IEEE Trans. Appl. Supercon., Vol. 19, No. 3, June 2009, pp.1356-13.

[6] M. A. Tartaglia, et al., "Summary of HINS Focusing Solenoid Production and Tests," IEEE Trans. Appl. Supercon., Vol. 20, No. 3, June 2010, pp.312-315.

[7] E. J. DiMarco, et al., "Certification of Superconducting Solenoid-Based Focusing Lenses," IEEE Trans. Appl. Supercon., Vol. 21, No. 3, June 2011, pp.1817-1820.

[8] T. H. Nicol, et al., "Design and Performance of Focusing Lenses for Installation into Superconducting Cryomodules of a Proton Linac," IEEE Trans. Appl. Supercon., Vol. 21, No. 3, June 2011, pp.1821-1824.

[9] G. Davis, et al., "HINS Linac SS-1 Section Prototype Focusing Solenoid Design," TD-08-010, FNAL, March 2008.

[10] G. Davis, et al., "HINS Linac SS2 Section Prototype Focusing Lens Design," TD-09-003, FNAL, March 12, 2009.

[11] I. Terechkine, "Quench Protection Study for Focusing Solenoids of Superconducting Sections of HINS Linac," TD-09-002, Feb. 12, 2009.

[12] S. Obraztsov, I. Terechkine, "HINS Front End Superconducting Focusing Solenoids - A Tool for Modeling Quench Propagation and Related Protection Issues," TD-06-063, FNAL, Dec. 07, 2006.

[13] I. Terechkine, V. Veretennikov, "Normal Zone Propagation in Superconducting Focusing Solenoids and Related Quench Protection Issues," IEEE Trans. Appl. Supercon., Vol. 18, No. 2, June 2008, pp.1325-1328.

[14] I. Terechkine, "SS2 Focusing Solenoid Quench Protection Study," TD09-016, FNAL, June 4, 2009.

[15] E. Khabiboulline and I. Terechkine, "Modeling Quench Propagation in Inductively Coupled System of Coils: Dump Resistors Connected in Parallel to Each Coil in the System," TD-11-011, Aug. 22, 2011.

[16] E. Khabiboulline and I. Terechkine, "SSR2 Focusing Lens Quench Protection Study: Dump Resistors Connected in Parallel to Coils of the Lens," TD-11-006, Apr. 06, 2011.

[17] E. Khabiboulline and I. Terechkine, "Quench Protection of SSR-2 Focusing Lens: Resistors Connected to All Coils of the Lens," TD-11018, Sep. 26, 201.

[18] J. DiMarco, et al., "Test Results for HINS SSR2 Focusing Lens," TD12-017, FNAL, October 2, 2012.

[19] S. Nagaitsev, et al., "PXIE: Project X Injector Experiment," Proceedings of IPAC2012, THPPP058, May 2012. 